\title{
Breastfeeding as a Tool that Empowers Infant Immunity Through Maternal Vaccination
}

Saad Musbah Alasil ${ }^{1}$ and Prameela Kannan Kutty ${ }^{2}$

${ }^{1}$ Department of Microbiology, Faculty of Medicine, MAHSA University, 59100 Kuala Lumpur, Malaysia

${ }^{2}$ Department of Paediatrics, Faculty of Medicine, MAHSA University, 59100 Kuala Lumpur, Malaysia

"Corresponding author: Prameela Kannan Kutty, Department of Paediatrics, Faculty of Medicine, MAHSA University, 59100 Kuala Lumpur, Malaysia, Tel: +6012-3220967; Fax: +603-7960799; E-mail: prameela.kutty@yahoo.com

Received date: 17 December 2014; Accepted date: 11 February 2015; Published date: 16 February 2015

Copyright: (c) 2015 Alasil SM, et al. This is an open-access article distributed under the terms of the Creative Commons Attribution License, which permits unrestricted use, distribution, and reproduction in any medium, provided the original author and source are credited.

\begin{abstract}
The dawn of maternal vaccination is an important milestone in breastmilk immunity. Breastmilk per se has immunopotential that protects the infant from important childhood diseases both in the immediate neonatal period and in the long term. Its immune nutritive attributes confer this exclusive early nourishment a cutting edge in defence that no other human nutrient can yet offer. Evidence that breastmilk is important in maturing the naiveté immune system and its potential to differentiate commensal and pathogenic microbes as well as its antimicrobial action are briefly reviewed. The vulnerability of the neonatal period to diseases and the increasing incidence of antibiotic resistance to pathogens must prompt us to continue to seek avenues through which primary disease preventive strategies can be emphasized and indeed improved. The advent of maternal vaccination has made it necessary that review and much research are both needed for elucidating the enrichment of the immune potential in breastmilk and how, because of it, some focused protective immunological responses may be triggered by further empowering the immunological potential of breastmilk constituents. . Primary disease prevention is achieved both by breastfeeding and by vaccination. We review here how the usefulness of breastfeeding and maternal vaccination may further empower breastmilk immunology.
\end{abstract}

Keywords: Breastfeeding; Breastmilk; Infant; Immunity; Maternal vaccination

\section{Introduction}

Naturally, breastmilk is the optimal nutrition for any infant. The ease of its digestion has a number of advantages to the infant who may utilise this conserved energy for rapid growth and a spectrum of important associated biological processes [1]. Human breastmilk also contains numerous protective factors against infectious diseases and potentially influences the development of the yet immature and developing infant immune system [2]. If the development of the immune system is significantly improved and prudently 'directed' with the introduction of the components of breastmilk, it then stands to reason that premature discontinuation of breastfeeding may predispose to chronic diseases in later life [3]. The improper development of the immune system is multifactorial and may be asymptomatic for several years. There is evidence linking the lack of breastfeeding with the pathogenesis of immune dysregulation such as autoimmune disorders and atopic allergies [2]. The early positive influences of human breast milk may be an essential protection against chronic disease in later life [2].

The infant's gastrointestinal tract encounters many foreign antigens on a daily basis [4]. Therefore, the mucosal surfaces face a constant dilemma on how to tolerate commensal microorganisms and harmless antigens while developing an immune response against pathogens. Therefore, the infant's mucosal immune system has to balance its responses depending on whether the antigen is pathogenic or not.

A review by Walker explores the hypothesis that the human neonatal gut at birth is immature, and that breastmilk contains functional nutrients that help provide the microenvironment for gut protection and maturation [5]. The author made the observation that breastmilk is the gold standard for protective nutrients to newborn infants and present clinical evidence of its strong protective effect against age-related infectious gastroenteritis [5]. Moreover, the author concluded that besides providing passive protection for the infant's immature mucosal immune system, breastmilk can also actively stimulate overall development of neonatal immunological processes.

\section{Breastmilk has a Role in Usefully "Directing" The Developing Immune System}

Breastmilk channels infant immunity in the right direction and evidence shown by past and recent studies indicate the proper immune direction conferred by early and exclusive breastfeeding and the emergence of immune dysregulation due to its absence. We review the function of directing the immune response provided by the practise of breastfeeding because maternal vaccine-mediated immunity in breastmilk can further enhance the innate function of breastmilk.

Breastfeeding provides the infant with the necessary balance that aims to maintain tissue homeostasis so that the mucosal tissue is able to perform its normal physiological functions [4]. It has been reported that breastmilk enables infants to acquire passive immunity via immunoglobulins and other antimicrobial compounds. It is also thought that breastfeeding have a beneficial impact on the intestinal microbial flora, improving the immunity to intestinal infections and reducing the likelihood of developing inflammatory diseases (Figure 1) [4]. The mechanism by which breastfeeding may have a beneficial effect on certain conditions such as inflammatory bowel disease (IBD) is possibly multifactorial [6]. It has been reported that breastfeeding is 
important for acquiring oral tolerance to microflora and food antigens $[7,8]$ which may prevent the development of IBD [8].

Furthermore, exclusive breastfeeding during the first 3 months of life protects children against allergic rhinitis [9]. A study by Hanson et al. suggested that breastfeeding facilitates immunological tolerance, and may thus decrease future risk of autoimmune disorders [10].

While the triggers to optimal immune development of the infant immune system are conceivably multifaceted, It is known that breastmilk's constituents supported the development of infant's immune system including promoting important events such as tolerance and priming of immunity [11]. If the size of thymus were taken to provide evidence of T cell development, an elegant study by Hasselbalch et al. measured the thymic index size and found that at 4 months of age, infants who were breastfed exclusively had significantly larger thymus glands than those who were partially breastfed or formula fed only [12]. The role of thymus in the development of the Tcell types indicates that breastfeeding has a direct effect on the maturation of infant's immune system.

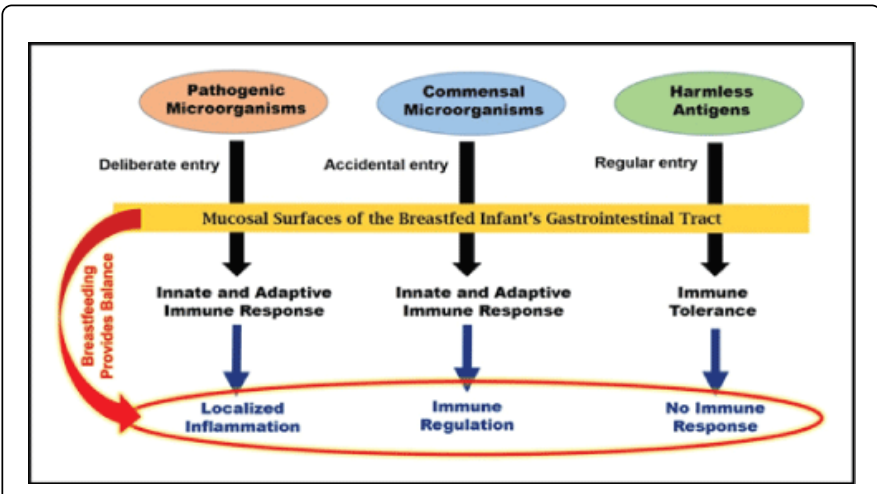

Figure 1: The response balance provided by breastfeeding that simultaneously allows effective pathogen clearance and maintains homeostasis in healthy tissue. Could this form of immune regulation be further enhanced by maternal vaccination?

\section{Breastmilk Components Empower Infant Immunity}

The immunological "power house" within breastmilk possesses important targets that can be used by maternal vaccination to transfer beneficial and focused vaccine-induced protection to the breast-fed infant while these breastmilk constituents themselves provide an innate protection to the infant specifically and non-specifically against diseases.

A living nutrient like blood, its antibodies confer defence against every disease the mother has ever been exposed to providing a dynamic maternal immune protection nurtured and transmitted to the suckling offspring. Akin to blood, early colostrum contains leukocytes such as macrophages and neutrophils that engulf microbial pathogens $[2,11,13]$. In addition, breastmilk contains $\mathrm{T}$ lymphocytes, natural killer cells and antibody-producing B lymphocytes that seem to survive the biochemical onslaught of passage through the infant's gastrointestinal system where they are absorbed into circulation and influence the immune response [13]. This contributes to the findings that breast-fed infants have lower incidences of important childhood diseases, including certain types of cancers [1,2]. Furthermore, an interesting link between the health status of the mother, infant and breastmilk through leukocyte levels have been suggested [14].

We review the bioactive components of breastmilk because maternal vaccine-mediated immunity can further enhance the antimicrobial activity of breastmilk's components (Table 1). The diverse mixture of bioactive components in human breastmilk may not only be important in sustaining defence against a spectrum of pathogens that abruptly challenge the immune system during extrauterine life but also has ingredients that provide the opportunity for the naiveté immune system to mature optimally and selectively as well as nurturing health-promoting micro mileu that are pivotal in improving the infant's immune status. Such optimal balances may additionally improve vaccine-induced immunological responses [15]. It has been reported that several non-specific factors in human breastmilk have antimicrobial effects [16]. Current knowledge explored how the diverse ingredients of human breastmilk promote immune development, facilitate tolerance and regulate the inflammatory responses of infants [15].

A variety of mechanisms are involved in the regulation of allergy development in infants through maternal immunization with allergens [14]. It has been proposed that the transfer of antigens, antibodies, and cytokines via breastfeeding will subsequently induce phenotypic alterations and regulatory populations in the infant's lymphocytes [14]. However, these mechanisms are still not completely understood. A study of mothers undergoing immunotherapy for two years prior to gestation revealed that immunized mothers passively transferred high amounts of allergen-specific IgG antibodies to their children compared with non-immunized mothers [17].

In a recent review, it was demonstrated that allergen-specific IgG and $\operatorname{IgE}$ antibodies with similar specificity are present and transferred from the blood of mothers into their breastmilk [18]. This opens the possibility to study the transmission of allergen-specific IgG antibodies from mothers to their offspring on the development of allergic sensitization in birth cohorts.

It has been reported that unique components of breastmilk confer both passive and active immune responses to the suckling infant [19]. Therefore, there has been consolidate firm efforts towards continuous breastfeeding in infants for up to two years [19]. In humans, IgG antibodies are transferred through the placenta from mothers to infants [20]. Since these maternal antibodies result from thymusdependent immune responses, they reflect maternal immunological experience [21]. Maternal antibodies not only confer passive immunity to the infant but also active stimulation of isotype expression and enhancement of early primary antibodies [21]. It is also believed that through regulation of host gene expression, maternal antibodies potentially provide enduring benefits to the intestinal immune system of the breast-fed infant which can persist into adulthood [17].

The prime defensive attribute in breastmilk is that it is linked to minimal inflammation via its abundant secretory immunoglobulin A ( $\operatorname{sgA})$ that do not activate the complement cascade [2]. Through immune exclusion, sIgA protects the intestinal lumen and respiratory passages from pathogenic microorganisms prior to first contact with epithelial receptors and may nullify bacterial virulence factors promoting a robust trigger of protection via gut-associated lymphoid tissue [22]. A study by Kassim et al. analysed breastmilk samples and sera from mother-infant pairs for specific antibodies against four bacterial pathogens [23]. Their results revealed that the mean levels of IgA to the four organisms were higher in the breastmilk than in both 
maternal and infant sera which may indicate a protective role for breastmilk against infections in early childhood [23].

Whereas much of the attention on maternal vaccination has been focused on vaccine-induced maternal IgG, IgA and IgM responses in breastmilk, IgD has not been explored in depth yet. Many features of IgD make it a potential target of maternal vaccination as it contributes to the immune defence against respiratory pathogens such as Haemophilus influenzae and Moraxella catarrhalis that are common neonatal infections [21]. It has been suggested that maternal rubellaspecific IgD persists longer than IgM and $\operatorname{IgA}$ after infection, and significant amounts of rubella-specific IgD can be transferred across the placenta during pregnancy [22]. The production of $\operatorname{IgD}$ is positively regulated byTH2 cytokines which allows IgD-induced vaccines to be more compatible with pregnancy than vaccines whose induction and protection require a strong pro-inflammatory TH1 environment [24]. Moreover, IgD inhibit IgE-induced histamine release but not cytokine production by basophils making it a target by maternal vaccination to control the rising rate childhood allergies without triggering adverse pregnancy outcomes associated with histamine [25].

A number of immunological factors such as cytokines have been identified in human breastmilk [26]. Cytokines that are present in breastmilk can potentially interact with mucosal and lymphoid tissues in the upper parts of the respiratory as well as gastrointestinal tract [26]. Breastmilk contains anti-proteases that interfere with proteolysis, and some digestive agents are not fully developed in the newborn period. Thus, biological functions of cytokines that are developmentally delayed in their production may be replaced by those present in breastmilk [26].

The potential roles of cellular immunity should be investigated in view of maternal vaccination during pregnancy particularly in colostrum where high amount of activated CD4+ $\mathrm{T}$ cells are encountered in comparison to peripheral blood suggesting selective migration to breastmilk [8]. However, little is known about the role of cellular immune response and their effect on breastmilk after maternal vaccination. There is an increased recognition in the protection provided by breastmilk's non-neutralising antibodies among HIVinfected mothers [16]. It has been reported that only one third of infants born to HIV-1 infected mothers who are continuously exposed to maternal breastmilk get infected. This observation raises the possibility that immune factors in infected women play a role in limiting HIV-1 transmission [27]. Moreover, a recent study by Mabuka et al. observed a robust non-neutralizing activity in breastmilk that was associated with infant infection status. Their study adds to the growing evidence of a potential role of non-neutralizing antibodies in limiting HIV-1 transmission and calls for more attention to the HIV-1 response [27].

There has been a lot of controversy concerning the physiological significance of complement system in human breastmilk. This is mainly due to the observation that breastmilk contains predominantly non-inflammatory as well as anti-inflammatory factors, while simultaneously protecting the infant against a wide range of diseases [28]. A study by Ogundele assessed the role of complement system to the bactericidal activity of human colostrum. Results proved that deposition of activated $\mathrm{C} 3$ fragments on the killed examined bacteria was demonstrated and that the bactericidal activity of breastmilk was completely inhibited by heat inactivation of complement. These observations suggests a pivotal role of breastmilk's complement system in protecting both the mammary gland and nursing infant against various pathogens [28].

Other components that appear to play an equally important role are oligosaccharides, glycoproteins, glycolipids [29], haptocorrin, lactoperoxidase, $\alpha$-lactalbumin, bile salt-stimulated lipase, $\beta$-casein, and tumour growth factors $[25,30]$. A review by Peterson et al. emphasized on the protective mechanisms of breastmilk's glycoconjugates, with a particular focus on the glycan moieties playing a role in protection against various diseases [29]. It is important to know that breastmilk contains all these factors against specific type of microorganisms. We hypothesize that maternal vaccination through the practice of breastfeeding may enhance the inherited antimicrobial activity of breastmilk's components.

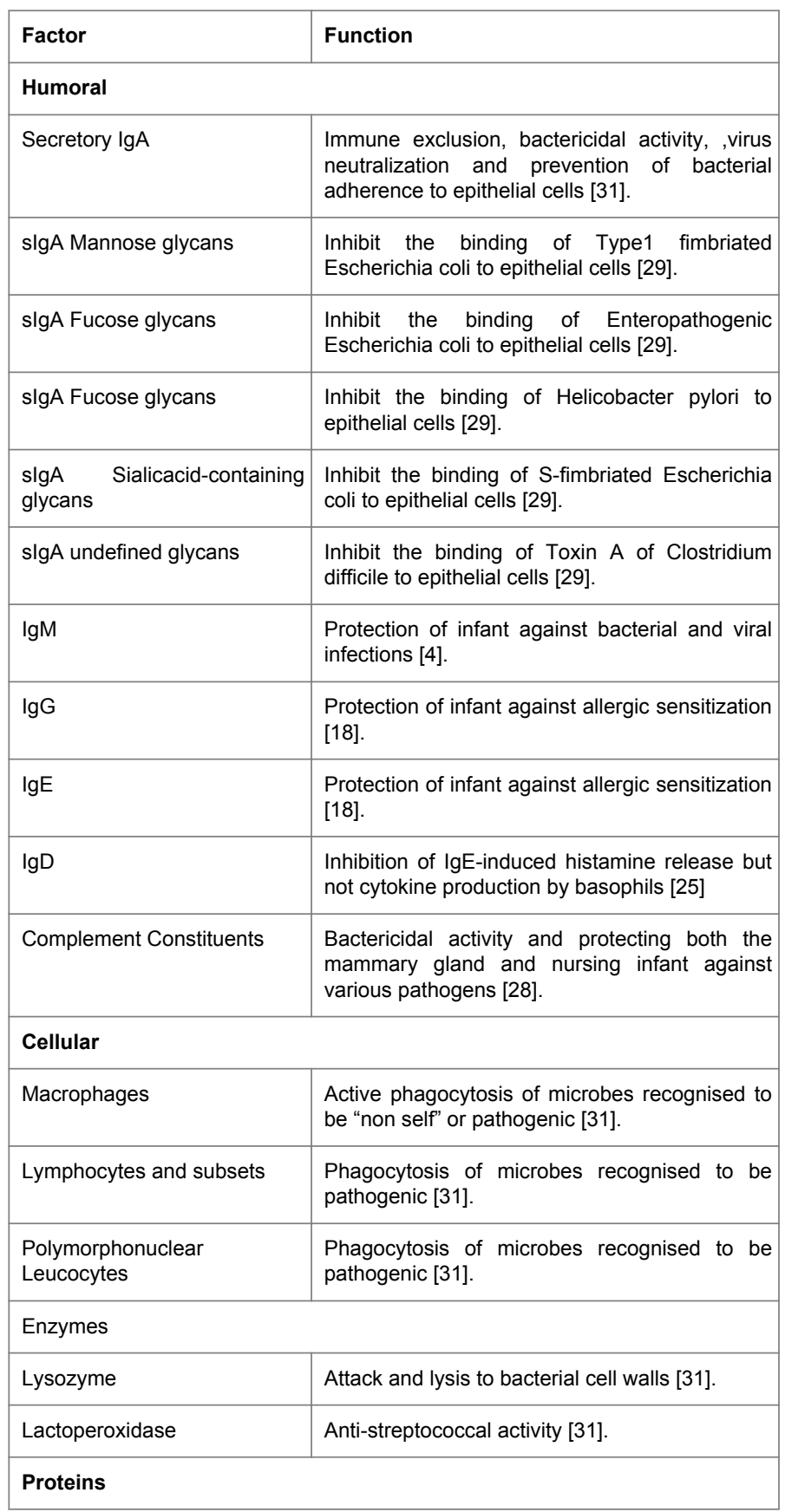




\begin{tabular}{|l|l|}
\hline Lactoferrin & $\begin{array}{l}\text { Inhibited Enterotoxigenic Escherichia coli } \\
\text { induced hemaglutination of human } \\
\text { erythrocytes in vitro [31]. }\end{array}$ \\
\hline Lactadherin & Inhibits the replication of rotaviruses [31]. \\
\hline Erythropoietin & $\begin{array}{l}\text { Effects on the integrity of infant's gut epithelia } \\
{[4] .}\end{array}$ \\
\hline Mucin & $\begin{array}{l}\text { Inhibit the binding of pathogenic bacteria and } \\
\text { viruses to buccal epithelial cells [29]. } \\
\text { Hypothesised to strengthen mucosae against } \\
\text { injury caused by specific parasitic infections } \\
{[29] .}\end{array}$ \\
\hline
\end{tabular}

\section{Other molecules}

Transforming Growth Factor Mediate anti-inflammatory responses [4]. (TGF- $\beta$ )

Interleukin-1 and Interleukin-8 Resistance to certain digestive processes [26].

Nerve Growth Factor, Insulin- Hypothesised non-specific anti-parasitic like Growth Factor, Tumor protection [30].

Necrosis Factor (TNF- $\alpha$ ) and Fibroblast Growth Factor

Enhances mucosal barriers and decreases incidence of some neonatal diseases such as necrotising enterocolitis $[28,30,32]$.

Table 1: Known and hypothesised protective factors in breastmilk, could these factors be augmented by maternal vaccination?

\section{Maternal Vaccination Strengthens Breastmilk Immunity}

In developing countries, the administration of live vaccines during pregnancy is contraindicated because of the possible transplacental transmission of the attenuated virus to the foetus [3]. In contrast vaccination with killed or inactivated virus has not shown to cause any risk to the fetus when administered during pregnancy [33]. This strategy has the potential to protect the foetus and the pregnant woman from many infectious diseases during pregnancy. In addition it provides better protection to the infant after birth during the first months of life when they are too young to be vaccinated [34].

The direction provided through the practice of breastfeeding towards infant immunity and the augmentation by breastmilk of infant immunity is still insufficient to drastically reduce the morbidity and mortality of many diseases among infants. It is known that vaccination confers protection by means of enhanced and focused immune responses [35]. It has been postulated that greater specific protection and more enduring immune responses transferred to the child through breastfeeding by maternal vaccination are of immense benefit to the infant. The many vaccine-induced immunological reactions may be assumed to be theoretically additive or even synergistic to the innate immune protection that is already present in breastmilk.

Many pathogens infect infants via mucosal surfaces. Therefore, stimulation of a local immune response at the site of infection would be the ultimate goal of a mucosal vaccine [31]. However, mucosal immunization faces difficulties in stimulating long-lasting immune responses. Therefore, there is a need to develop effective vaccine adjuvants and vaccine delivery systems that transport antigen into the mucosa-associated lymphoid tissue (MALT) and provide protective immunity [31]. It is believed that breastmilk through the act of exclusive breastfeeding provides the infant with such protective advantage.

In a study emphasising the role of breastfeeding as compliment to vaccination, it was found that breastfeeding seems to have a positive overall effect on vaccination [35]. The role of breastfeeding influencing different aspects of vaccination in the first 6 months which is a period of dynamic pain management, immunomodulation of vaccine responses and metabolism of thimerosal-such affects were looked at [36]. The study indicated that breastfeeding is a positive factor in mothers and infants coping with the stressful situations linked to parenteral vaccines, improves responses to vaccines and even modifies the effect on metabolism of ethyl mercury derived from some vaccines [36]. Optimal protection and health promotion supporting vaccination must highlight early initiation and sustenance of breastfeeding for six months [36].

A prospective study by Pisacane et al. was conducted at a paediatric vaccination center in Italy to evaluate the effects of breastfeeding on the risk for fever after routine immunizations [37]. Results showed that the protection conferred by breastfeeding persisted even when considering the role of several potential confounders [37]. The authors concluded that breastfeeding was associated with a decreased incidence of fever after immunizations.

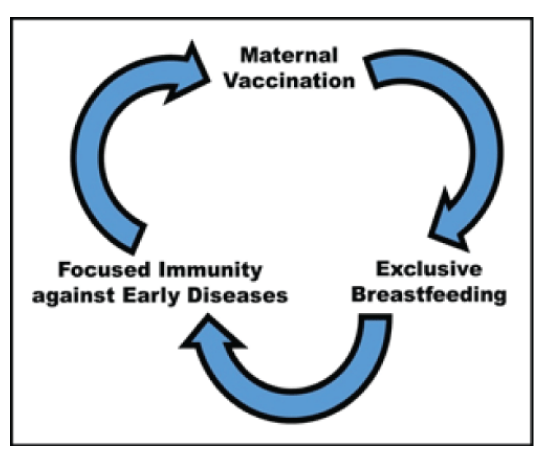

Figure 2: Maternal vaccination improves breastmilk protection while early neonatal diseases require augmented breastmilk protection enabled by maternal vaccination.

Although breastfeeding is essential to modulate immune defences, vaccines are crucial to prevent infectious diseases. However, maternal factors related to breastmilk constituents and feeding practices can affect the response to vaccines [38]. A study by Dórea indicated that maternal constitutional and environmental factors and feeding practices can affect the response to vaccines. Sucklings without receiving the full benefit of breastfeeding could have altered immune responses affecting vaccine outcome [38]. The study concluded that vaccination practices should encourage the practice of exclusive breastfeeding [38].

Maternal vaccination during pregnancy results in an augmentation of disease-specific maternal antibodies. Immunoglobulin G (IgG) is mainly transferred through the placenta during the third trimester of pregnancy, while secretory IgA is passed through breastmilk. Newborns are partially protected against infections by these antibodies [34]. The placental transport of IgG depends on the placental function and on the concentration of maternal antibodies in the pregnant woman [7]. The concentration of IgG in women at childbearing age is defined by the previous exposure to the antigen through either disease 
or vaccination. A study by Gall et al. demonstrated that increased titres of maternal IgG antibodies against diphtheria and pertussis antigens have been described after vaccination during pregnancy which may prevent neonatal pertussis infection [6]. In contrast, little is known about the effect of vaccination during pregnancy on the transfer of vaccine-induced maternal secretory IgA via breastfeeding.

A recent review provides an overview of the effect of vaccination during pregnancy on the immunological protection of the newborn [34]. The review emphasized the beneficial effects of maternal vaccination during pregnancy on the amount of disease-specific sIgA in breastmilk. To substantiate this observation, it was noted that breastfeeding after maternal vaccination resulted in a lower incidence of some infectious disease in infants [34]. Further research is needed to assess the effect on disease-specific sIgA in breastmilk and its potentially beneficial effects to the infant.

A review by Mantis et al. elegantly described many potential intrinsic biological activities of sIgA such as blocking attachment by steric hindrance and receptor domains, agglutination, entrapment and clearance and direct effects on bacterial virulence. Secretory IgA also functions as a part of the innate immunity being independent of antibody variable region and other plausible pathways for immune defence [39]. Furthering this description, it is suggested that while sIgA has a continuum of protective actions including that which has been described, sIgA is produced as a result of exposure to diseases and in greater magnitude as a consequence of maternal vaccination and its capacity may be augmented relevant to the clearance of pathogens or their products [39].

It has been reported that breastfeeding influences the infant's response to common childhood vaccinations [40]. The presence of maternal anibodies are known to interfere with infant vaccine responses. A study by Crowe Jr. explored the recent knowledge regarding the effect of maternal antibodies on respiratory virus and measles virus immunization, and it reviewed the current approaches to overcoming antibody-mediated immunosuppression [41].

Plausible explanations to how maternal antibodies dampen infant immune responses have been made. In a review by Siegrist, the mechanisms by which maternal antibodies inhibit the infant's vaccine responses were assessed [42]. This inhibition is B cell determinantspecific that depends on the ratio between Maternal Antibody $(\mathrm{Mat} A \mathrm{~b})$ titres at the time of immunization and the dose of vaccine antigens. The author suggested that neutralization of vaccine replication or Fc $\gamma$ RIIB-mediated inhibitory signalling to the infant's B cells would not account for these characteristics. In contrast, determinant-specific masking of $\mathrm{B}$ cell epitopes and the uptake of antigen presenting cells (APCs) to MatAb which is vaccine antigen immune complexes explains the pattern of responses to infant vaccines [42].

There is evidence from various studies suggesting that the cellular components in breastmilk survive the passage through the infant's digestive tract and are found in the draining lymph nodes [10,43]. The observation that these cells remain functional comes from positive skin responses to purified protein derivative (PPD) of mycobacteria which can be transferred from one individual to another via breastmilk [44]. Other studies have shown reductions in the alloreactivity of breast-fed infants who were given maternal allografts [45]. This study supports the idea that breastmilk's leukocytes are able to interact with the infant's intestinal mucosa leading to a tolerance to maternal antigens. The questions that we believe are important to be asked are these cells capable of amplifying immunity, focusing useful immunity and eliminating unnecessary immune reactions? Could these immune reactions be incorporated within vaccines? Currently hypothetical, these issues need consideration.

A study by Pabst et al. assessed the effect of breastfeeding on the immune response to BCG vaccination in infants who were vaccinated either at birth or at 1 month of age [46]. Their results showed that breastfeeding significantly enhanced cell-mediated immune response to BCG vaccine given at birth but had no significant effect if the vaccine was given after 1 month. These findings were not related to maternal history of tuberculosis or BCG vaccination [46].

Additionally, the timing of immunization to the infant seems to be important. A study by Levi et al. demonstrated that there was a relationship between the antibody titres of infants to the pertussisdiphtheria-tetanus antigens and the antibody response to the pertussis-diphtheria-tetanus vaccine [47]. These results suggested that certain antibody-antigen complexes are more immunogenic than antigens alone emphasizing that when infants are immunized shortly after birth in the presence of a large amount of circulating antibody the immune response is depressed [47].

Pneumococcal diseases such as pneumonia, septicaemia and bacterial meningitis are important to be prevented from birth as children younger than two years of age are extremely susceptible to these infections [48]. However, infant vaccination against pneumococcal diseases starts only as early as around two months of age and in many developing countries is not part of the compulsory vaccination schedule, making it more difficult to be implemented in some parts of the population [48]. Another study by Greenwood explored the potential of maternal immunization to prevent early infant deaths in developing countries. The author emphasized that the incidence of pneumococcal infections is high in many developing countries and a significant proportion of these infections occur at an age before vaccine induced protection [49].

A recent animal study provided evidence that maternal immunization with pneumococcal surface protein A protects against pneumococcal diseases among the offspring [50]. Moreover, immunization with recombinant PspA and cholera toxin B subunit (CTB) resulted in Anti-PspA specific IgG present at a high level in the serum and milk of immunized mothers as well as in the sera of their offspring [50]. Authors suggested that the rationale of maternal immunization pari passu continued exclusive breastfeeding is highly applicable in these diseases and is a preventive strategy to reduce morbidity and mortality during early childhood [50].

The potency and safety of vaccines administered to mothers and the seasonal influence of microbes that are adept at antigenic variations are immunological characteristics that must be considered in the administration of vaccination to pregnant mothers.

Previous studies explored the immunogenicity of inactivated influenza virus vaccine during the second and third trimesters of pregnancy [51]. The authors concluded that immunization of pregnant women can provide sufficient protection of the newborns via transplacental maternal antibodies if a more potent influenza vaccine is administered and the women deliver during the influenza season [51].

Another study provided seroepidemiological data on influenza A and $B$ antibodies in pregnant women and their offspring in Germany [52]. Results revealed that antibody titers against both influenza A and 
B viruses were significantly lower in mothers than in their newborns [52]. The authors concluded that because of active placental transport of IgG antibodies, neonates have higher prevalence and concentrations of influenza A and B virus-specific antibodies induced by natural infections than their mothers [52].

A study by Zaman at al. estimated the incidence of illness, incidence rate ratios and effectiveness of inactivated influenza vaccine administered during pregnancy of 340 mothers in Bangladesh [53]. The authors showed that inactivated influenza vaccine reduced the influenza illness by $63 \%$ in infants up to 6 months of age and prevented about a third of all febrile respiratory illnesses in mothers and young infants [53]. Authors concluded that maternal vaccination is a protective strategy of great benefits for both mothers and infants.

In a cohort study by Nankabirwa et al., the relationship between maternal education and vaccination status of infants was explored [54]. Results showed that women with secondary education were at least $50 \%$ less likely to miss their infants' scheduled vaccinations in comparison to women with only a primary school education. The authors recommend that resources for routine childhood vaccinations should specifically be targeted at women with low formal education [54]. We hypothesize that there are common areas of intersection that represent known and plausible beneficial effects of the tetrad of maternal vaccination, effective counselling on infant vaccination, breastfeeding and preventive counselling of diseases as primary health care tools (Figure 3).

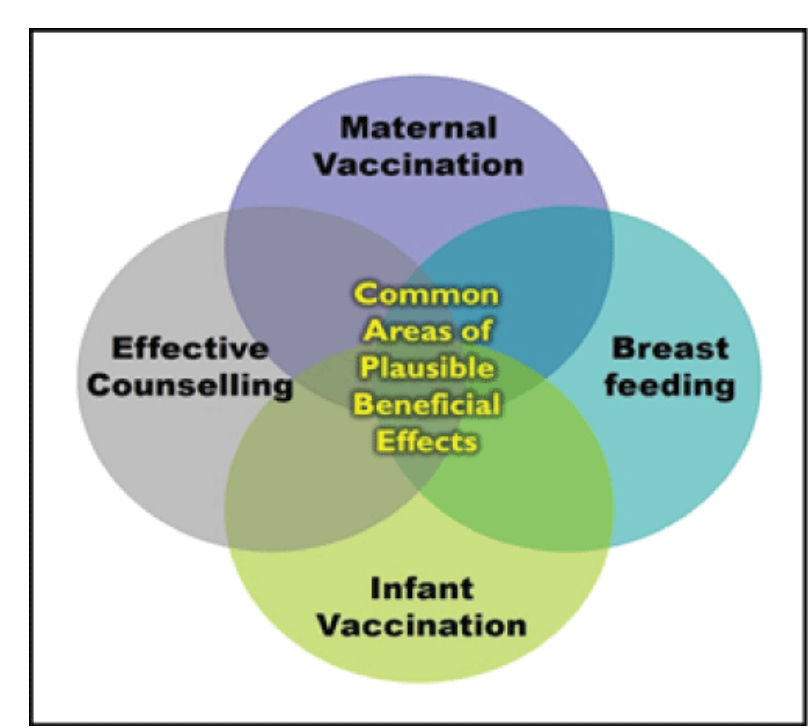

Figure 3: Common areas of plausible beneficial effects of maternal vaccination, effective counselling on infant vaccination, breastfeeding and preventive counselling of diseases.

\section{Conclusion}

There is justifiable evidence that breastfeeding plays an important role in the immune development of the naiveté immune system. It is known that maternal factors also contribute to the infant immune development. Early exposure to diseases or environment that impacts the mother's immunological experience such as her immunological milieu, some of the relevant innate and adaptive immune defences beneficial to her, the tolerance that she must achieve towards her immune environment and immunological memory may influence what she may pass on to her suckling infant.

An important property of breastmilk is its capacity to downregulate the immature and excessive inflammation in newborns. It has been reported that many components of breastmilk are antiinflammatory in nature and that these components can work separately or together to control the immature inflammation until the mature immune response can develop. In the context of this article, this capacity is deemed to be beneficial in dampening unnecessary immune responses due to either infant or maternal vaccination and may explain some of the decreased side effects observed during vaccination of a breastfed infant.

The immunological components in breastmilk are many. There are diverse and multifunctional. It is proposed that vaccines that are given to the mother can pass through breastmilk and potentially utilise these breastmilk components to augment action and protection. Protection via maternal vaccination is vital during a time that some important vaccines may not yet be administered by schedule to the neonate or because desired vaccine responses in the neonate are difficult to elicit due to physiologic immaturity of the neonatal immune system.

The increasing global incidence of antibiotic resistance to treat important infections affecting the infant makes it crucial to emphasize the importance of breastfeeding and possibly enhancing microbial protection transferred through maternal vaccination as a primary preventive strategy. Responses during infancy by maternal vaccinations obtained through breastfeeding need to be further explored in the light of their protective efficacy, duration of protection and even comprehensive action vis-à-vis benefits to the immune system for a broader range of protection. It is hypothesised that many collateral benefits may be obtained by maternal vaccination together with breastfeeding such as the nonspecific general augmentation of breastmilk immunity and even long term protection.

It is felt that globally, the more accessed antenatal clinics should routinely practice the delivery of this multi-pronged system of primary protection augmenting primary prevention through the tetrad of maternal vaccination, advice on infant vaccination, exclusive breastfeeding and general health counselling of the prevention of communicable and non-communicable diseases (Figure 3). After further exploration and observational study, governments and health care providers could plausibly save revenue on the treatment and care of infectious diseases and even other diseases in the long term, using the primary preventive tetrad, given the enormous immunological prowess that lies within breast milk.

This review reinforces the concept that high titers of maternal antibodies against bacterial and viral antigens are transferred to the fetus when women are vaccinated in the antenatal period. If a program of maternal vaccination in the second trimester were to be adopted as a standard practice, there would be less risk for infants to various infectious diseases in the first six months of life until their vaccination establishes active immunity.

Maternal vaccination has emerged as a promising approach to prevent morbidity among infants. Considerable achievements have been made in the past decade, with a number of vaccines being recommended for pregnancy. However, the public acceptance of maternal vaccination has been low in many countries. Besides the ethical, legal and socioeconomic restraints, significant gaps exist in our knowledge of the efficacy and safety of maternal vaccines in pregnant women and those susceptible to high-risk pregnancies, and no 
maternal vaccines against a large number of old and emerging pathogens are available. To tackle these scientific challenges, scientists and clinicians from various disciplines must work in tandem guided by a holistic understanding of the maternal, fetal and neonatal immunologic responses to vaccines and how such responses may be transmitted or augmented by the practise of exclusive breastfeeding to the breastfed infant. The various topics in our review are intended for such a purpose.

\section{Acknowledgment}

This study was supported by MAHSA University. The authors would like to thank the Faculty of Medicine at MAHSA University who assisted in providing all the logistics needed to complete this review. The authors had full access to all the data in the study and take responsibility for the integrity of the data and the accuracy of the data analysis. The authors declare that there was no financial disclosure and that they have no conflict of interest.

\section{References}

1. Fredregill S, Fredregill R (2010) The Everything Breastfeeding Book. The helpful, reassuring adviceand practical information you need for a comfortable-pand confident-nursing experience. (2nd Edn), Adams Media, Massachusetts, USA.

2. Jackson KM, Nazar AM (2006) Breastfeeding, the immune response, and long-term health. J Am Osteopath Assoc 106: 203-207.

3. Bozzo P, Narducci A, Einarson A (2011) Vaccination during pregnancy. Can Fam Physician 57: 555-557.

4. Williams AE (2012) Immunology Mucosal and Body Surface Defences. Wiley-Blackwell, UK.

5. Walker A (2010) Breast milk as the gold standard for protective nutrients. J Pediatr 156: S3-7.

6. Gall SA, Myers J, Pichichero M, (2011) Maternal immunization with tetanus- diphtheria-pertussis vaccine: effect on maternal and neonatal serum antibody levels. Am J Obstet Gynecol 204: 334-335.

7. Linder N, Tallen-Gozani E, German B, Duvdevani P, Ferber A, et al. (2004) Placental transfer of measles antibodies: effect of gestational age and maternal vaccination status. Vaccine 22: 1509-1514.

8. Peroni DG, Chirumbolo S, Veneri D, Piacentini GL, Tenero L, et al. (2013) Colostrum-derived $\mathrm{B}$ and $\mathrm{T}$ cells as an extra-lymphoid compartment of effector cell populations in humans. J Matern Fetal Neonatal Med 26: 137-142.

9. Mimouni Bloch A, Mimouni D, Mimouni M, Gdalevich M (2002) Does breastfeeding protect against allergic rhinitis during childhood? A metaanalysis of prospective studies. Acta Paediatr 91: 275-279.

10. Hanson LA, Korotkova M, Håversen L, Mattsby-Baltzer I, Hahn-Zoric $\mathrm{M}$, et al. (2002) Breast-feeding, a complex support system for the offspring. Pediatr Int 44: 347-352.

11. Goldman AS (1993) The immune system of human milk: antimicrobial, antiinflammatory and immunomodulating properties. Pediatr Infect Dis J 12: 664-671.

12. Hasselbalch H, Jeppesen DL, Engelmann MD, Michaelsen KF, Nielsen MB (1996) Decreased thymus size in formula-fed infants compared with breastfed infants. Acta Paediatr 85: 1029-1032.

13. Wold AE, Adlerberth I (1998) Does breastfeeding affect the infant's immune responsiveness? Acta Paediatr 87: 19-22.

14. Victoe JR (2014) Influence of Maternal Immunization with Allergens on the Thymic Maturation of Lymphocytes with Regulatory Potential in Children: A Broad Field for Further Exploration. Journal of Immunology Research 1-6.

15. Field CJ (2005) The immunological components of human milk and their effect on immune development in infants. J Nutr 135: 1-4.
16. Hanson L, Silfverdal SA, Strömbäck L, Erling V, Zaman S, et al. (2001) The immunological role of breast feeding. Pediatr Allergy Immunol 12 Suppl 14: 15-19.

17. Flicker S, Marth K, Kofler H, Valenta R (2009) Placental transfer of allergen-specific IgG but not IgE from a specific immunotherapy-treated mother. Journal of Allergy and Clinical Immunology 124: 1358-1360.

18. Hochwallner H, Alm J, Lupinek C, Johansson C, Mie A, et al. (2014) Transmission of allergen-specific IgG and IgE from maternal blood into breast milk visualized with microarray technology. J Allergy Clin Immunol 134: 1213-1215.

19. Prameela KK, Mohamed AK (2010) Breast milk immunoprotection and the common mucosal immune system: a review. Malays J Nutr 16: 1-11.

20. Palmeira P, Quinello C, Silveira-Lessa AL, Zago CA, Carneiro-Sampaio M (2012) IgG placental transfer in healthy and pathological pregnancies. Clin Dev Immunol 2012: 985646.

21. Chen $\mathrm{K}, \mathrm{Xu} \mathrm{W}$, Wilson $\mathrm{M}, \mathrm{He} \mathrm{B}$, Miller NW, et al. (2009) Immunoglobulin $\mathrm{D}$ enhances immune surveillance by activating antimicrobial, proinflammatory and B cell-stimulating programs in basophils. Nature Immunology 10: 889- 898.

22. Faucette AN, Unger BL, Gonik B, Chen K (2015) Maternal vaccination: moving the science forward. Hum Reprod Update 21: 119-135.

23. Kassim OO, Raphael DH, Ako-Nai AK, Taiwo O, Torimiro SE, et al. (1989) Class-specific antibodies to Bordetella pertussis, Haemophilus influenzae type b, Streptococcus pneumoniae and Neisseria meningitidis in human breast-milk and maternal-infant sera. Ann Trop Paediatr 9: 226-232.

24. Levan-Petit I, Lelievre E, Barra A, Limosin A, Gombert B, et al. (1999) $\mathrm{T}(\mathrm{h}) 2$ cytokine dependence of $\mathrm{IgD}$ production by normal human B cells. Int Immunol 11: 1819-1828.

25. Cerutti A (2010) Methods for treating IgE-mediated disorder. Cornell University, USA.

26. Garofalo R (2010) Cytokines in human milk. J Pediatr 156: S36-40.

27. Mabuka J, Nduati R, Odem-Davis K, Peterson D, Overbaugh J (2012) HIV-specific antibodies capable of ADCC are common in breastmilk and are associated with reduced risk of transmission in women with high viral loads. PLoS Pathog 8: e1002739.

28. Ogundele MO (1999) Complement-mediated bactericidal activity of human milk to a serum-susceptible strain of E. coli 0111. J Appl Microbiol 87: 689-696.

29. Peterson R, Cheah WY, Grinyer J, Packer N (2013) Glycoconjugates in human milk: protecting infants from disease. Glycobiology 23: 1425-1438.

30. Kutty PK (2014) Breastfeeding and risk of parasitic infection-a review. Asian Pacific Journal of Tropical Biomedicine 4: 847-858.

31. Chapel H, Haeney M, Misbah S, Snowden N (2014) Essentials of Clinical Immunology. (6th Edn), Wiley Blackwell, United Kingdom.

32. Cummins AG, Thompson FM (2002) Effect of breast milk and weaning on epithelial growth of the small intestine in humans. Gut 51: 748-754.

33. Moro PL, Museru OI, Broder K, Cragan J, Zheteyeva Y, et al. (2013) Safety of influenza A (H1N1) 2009 live attenuated monovalent vaccine in pregnant women. Obstet Gynecol 122: 1271-1278.

34. Maertens K (2014) Breastfeeding after maternal immunisation during pregnancy: providing immunological protection to the newborn: a review. Vaccine 32: 1786-1792.

35. Ogra PL1, Faden H, Welliver RC (2001) Vaccination strategies for mucosal immune responses. Clin Microbiol Rev 14: 430-445.

36. Dòrea JG (2009) Breastfeeding is an essential complement to vaccination. Acta Paediatr 98: 1244-1250.

37. Pisacane A, Continisio P, Palma O, Cataldo S, De Michele F, et al. (2010) Breastfeeding and risk for fever after immunization. Pediatrics 125: e1448-1452.

38. Dórea JG (2012) Breast-feeding and responses to infant vaccines: constitutional and environmental factors. Am J Perinatol 29: 759-775. 
Citation: Alasil SM, Kutty PK (2015) Breastfeeding as a Tool that Empowers Infant Immunity Through Maternal Vaccination. J Vaccines Vaccin

39. Mantis NJ, Rol N, Corthésy B (2011) Secretory IgA's complex roles in immunity and mucosal homeostasis in the gut. Mucosal Immunol 4: 603-611.

40. Hanson LA (1998) Breastfeeding provides passive and likely long-lasting active immunity. Ann Allergy Asthma Immunol 81: 523-533.

41. Crowe JE (2001) Influence of maternal antibodies on neonatal immunization against respiratory viruses. Clin Infect Dis 33: 1720-1727.

42. Siegrist CA (2003) Mechanisms by which maternal antibodies influence infant vaccine responses: review of hypotheses and definition of main determinants. Vaccine 21: 3406-3412.

43. Jain L, Vidyasagar D, Xanthou M, Ghai V, Shimada S, et al. (1989) In vivo distribution of human milk leucocytes after ingestion by newborn baboons. Arch Dis Child 64: 930-933.

44. Ogra SS, Weintraub D, Ogra PL (1977) Immunologic aspects of human colostrum and milk. III. Fate and absorption of cellular and soluble components in the gastrointestinal tract of the newborn. J Immunol 119: 245-248.

45. Campbell DA, Lorber MI, Sweeton JC, Turcotte JG, Niederhuber JE, et al. (1984) Breast feeding and maternal-donor renal allografts. Possibly the original donor-specific transfusion. Transplantation 37: 340-344.

46. Pabst HF, Godel J, Grace M, Cho H, Spady DW, et al. (1989) Effect of breast-feeding on immune response to BCG vaccination. Lancet 1: 295-297.

47. Levi MI, Kravtzov FE, Levova TM, Fomenko GA (1969) The ability of maternal antibody to increase the immune response in infants. Immunology 16: 145-148.
48. Nuorti JP, Whitney CG (2010) Prevention of Pneumococcal Disease Among Infants and Children --- Use of 13-Valent Pneumococcal Conjugate Vaccine and 23-Valent Pneumococcal Polysaccharide Vaccine: Recommendations of the Advisory Committee on Immunization Practices (ACIP). Centres for Disease Control and Prevention p 1-18.

49. Greenwood B (2003) Maternal immunisation in developing countries. Vaccine 21: 3436-3441.

50. Kono M, Hotomi M, Hollingshead SK, Briles DE, Yamanaka N (2011) Maternal immunization with pneumococcal surface protein A protects against pneumococcal infections among derived offspring. PLoS One 6: e27102.

51. Sumaya CV, Gibbs RS (1979) Gibbs, Immunization of pregnant women with influenza A/New Jersey/76 virus vaccine: reactogenicity and immunogenicity in mother and infant. J Infect Dis 140: 141-146.

52. Wutzler P, Schmidt-Ott R, Hoyer H, Sauerbrei A (2009) Prevalence of influenza $\mathrm{A}$ and $\mathrm{B}$ antibodies in pregnant women and their offspring. J Clin Virol 46: 161-164.

53. Zaman K, Roy E, Arifeen SE, Rahman M, Raqib R, et al. (2008) Effectiveness of maternal influenza immunization in mothers and infants. N Engl J Med 359: 1555-1564.

54. Nankabirwa V, Tylleskär T, Tumwine JK, Sommerfelt H; Promise-ebf Study Group (2010) Maternal education is associated with vaccination status of infants less than 6 months in Eastern Uganda: a cohort study. BMC Pediatr 10: 92. 